\title{
A CLASS OF INTEGRABLE POLYNOMIAL VECTOR FIELDS
}

Abstract. We study the integrability of two-dimensional autonomous systems in the plane of the form $\dot{x}=-y+X_{s}(x, y), \dot{y}=x+Y_{s}(x, y)$, where $X_{s}(x, y)$ and $Y_{s}(x, y)$ are homogeneous polynomials of degree $s$ with $s \geq 2$. First, we give a method for finding polynomial particular solutions and next we characterize a class of integrable systems which have a null divergence factor given by a quadratic polynomial in the variable $\left(x^{2}+y^{2}\right)^{s / 2-1}$ with coefficients being functions of $\tan ^{-1}(y / x)$.

1. Introduction. We consider two-dimensional autonomous systems of differential equations of the form

$$
\dot{x}=-y+X_{s}(x, y), \quad \dot{y}=x+Y_{s}(x, y),
$$

where

$$
X_{s}(x, y)=\sum_{k=0}^{s} a_{k} x^{k} y^{s-k}, \quad Y_{s}(x, y)=\sum_{k=0}^{s} b_{k} x^{k} y^{s-k}
$$

are homogeneous polynomials of degree $s$, with $s \geq 2$, and with $a_{k}$ and $b_{k}$, $k=0,1, \ldots, s$, being arbitrary real coefficients. Recently, these systems have been studied by several authors (see for instance [1], [3], [5], [6] and $[8]$ ), especially in order to obtain information about the number of small amplitude limit cycles and to determine the cyclicity of the origin (see for instance [4] and [7]). In this paper we study their integrability.

Our aim is to find solutions $W(x, y)=0$ of system $(1.1)$, where $W(x, y)$ is a null divergence factor (this notion will be defined below). In Theorem 1 we give an explicit method for obtaining such a factor, which is used in Theorem 2 to construct a particular class of integrable fields.

1991 Mathematics Subject Classification: Primary 34A05; Secondary 34C05. Key words and phrases: center-focus problem, integrable systems in the plane. Research partially supported by a University of Lleida Project/94. 
We can write system (1.1) (see [2]) in polar coordinates $x=r \cos \varphi$ and $y=r \sin \varphi$ as

$$
\dot{r}=P_{s}(\varphi) r^{s}, \quad \dot{\varphi}=1+Q_{s}(\varphi) r^{s-1},
$$

where $P_{s}(\varphi)$ and $Q_{s}(\varphi)$ are trigonometric polynomials of the form

$$
\begin{aligned}
P_{s}(\varphi)= & R_{s+1} \cos \left((s+1) \varphi+\varphi_{s+1}\right)+R_{s-1} \cos \left((s-1) \varphi+\varphi_{s-1}\right) \\
& +\ldots+ \begin{cases}R_{1} \cos \left(\varphi+\varphi_{1}\right) & \text { if } s \text { is even, } \\
R_{0} & \text { if } s \text { is odd, }\end{cases} \\
Q_{s}(\varphi)= & -R_{s+1} \sin \left((s+1) \varphi+\varphi_{s+1}\right)+\bar{R}_{s-1} \sin \left((s-1) \varphi+\bar{\varphi}_{s-1}\right) \\
& +\ldots+ \begin{cases}\bar{R}_{1} \sin \left(\varphi+\bar{\varphi}_{1}\right) & \text { if } s \text { is even, } \\
\bar{R}_{0} & \text { if } s \text { odd, }\end{cases}
\end{aligned}
$$

where $R_{i}, \bar{R}_{i}, \varphi_{i}$ and $\bar{\varphi}_{i}$ are real constants.

If we make the change of variable $R=r^{s-1}$, then system (1.2) becomes

$$
\dot{R}=(s-1) P_{s}(\varphi) R^{2}, \quad \dot{\varphi}=1+Q_{s}(\varphi) R .
$$

In the study and determination of the first integrals for quadratic systems and homogeneous cubic systems (see [2]), we used a technique consisting in the research of polynomial particular solutions of system (1.3) of the form

$$
V(R, \varphi)=1+V_{1}(\varphi) R+V_{2}(\varphi) R^{2}+\ldots+V_{p}(\varphi) R^{p}=0,
$$

where $V_{k}(\varphi), k=1, \ldots, p$, are homogeneous trigonometric polynomials of degree $k(s-1)$ in the variables $\cos \varphi$ and $\sin \varphi$. The main results are the following.

A function $W(x, y)$ will be called a null divergence factor for system (1.1) if $W(x, y)=0$ is a particular solution for this system and the divergence of the vector field

$$
C=\left(\frac{-y+X_{s}(x, y)}{W(x, y)}, \frac{x+Y_{s}(x, y)}{W(x, y)}\right)
$$

defined on $\mathbb{R}^{2} \backslash\{(x, y): W(x, y)=0\}$ is zero.

We notice that if the divergence of a vector field is zero then system (1.1) defined for this vector field is integrable. In particular, if system (1.1) has a null divergence factor then this system is integrable and the origin is a center.

By using the functions $x_{i}, i=1, \ldots, p$, defined implicitly by

$$
V_{1}=\sum_{j=1}^{p} x_{j}, \quad V_{2}=\sum_{\substack{j, k=1 \\ j<k}}^{p} x_{j} x_{k}, \quad \ldots, \quad V_{p}=x_{1} x_{2} \ldots x_{p}
$$

the function (1.4) can be written as $V(R, \varphi)=\prod_{i=1}^{p}\left(1+x_{i}(\varphi) R\right)$. 
THEOREM 1. If

$$
(V(R, \varphi))^{\alpha}=\left(\prod_{i=1}^{p}\left(1+x_{i}(\varphi) R\right)\right)^{\alpha}
$$

is a null divergence factor for system (1.1) with $\alpha$ a real number, then the functions $x_{i}(\varphi), i=1, \ldots, p$, satisfy the following system of differential equations:

$$
\frac{d x_{i}}{d z}=\frac{\frac{x_{i}}{z-x_{i}}}{\frac{s+1}{s-1}+\alpha \sum_{j=1}^{p} \frac{x_{j}}{z-x_{j}}}, \quad i=1, \ldots, p,
$$

where $z=Q_{s}(\varphi)$.

For $p=2$ it is possible to find the general solution for system (1.6) and therefore to determine a null divergence factor for system (1.1). In this case, we have

TheOREM 2. For $s \in \mathbb{N}$ with $s \geq 2$, and arbitrary $k_{1}, k_{2}, \varphi_{0} \in \mathbb{R}$, the system of the form (1.2) with

$$
\begin{aligned}
P_{s}(\varphi)= & 2\left(-k_{1} \cos ^{s-2}\left(\varphi+\varphi_{0}\right) \sin ^{3}\left(\varphi+\varphi_{0}\right)\right. \\
& \left.+k_{2} \sin ^{s-2}\left(\varphi+\varphi_{0}\right) \cos ^{3}\left(\varphi+\varphi_{0}\right)\right), \\
Q_{s}(\varphi)= & \left(k_{1} \cos ^{s-1}\left(\varphi+\varphi_{0}\right)-k_{2} \sin ^{s-1}\left(\varphi+\varphi_{0}\right)\right) \cos 2\left(\varphi+\varphi_{0}\right),
\end{aligned}
$$

is integrable.

In cartesian coordinates $x=r \cos \left(\varphi+\varphi_{0}\right)$ and $y=r \sin \left(\varphi+\varphi_{0}\right)$, we can write system (1.7) in the form

$$
\begin{aligned}
& \dot{x}=-y-k_{1} x^{s-1} y+k_{2} y^{s-2}\left(2 x^{2}-y^{2}\right), \\
& \dot{y}=x+k_{1} x^{s-2}\left(x^{2}-2 y^{2}\right)+k_{2} x y^{s-1},
\end{aligned}
$$

with $s \in \mathbb{N}$ and $s \geq 2$. We notice that the origin is a center for system (1.8).

In Section 2 we give a method of obtaining particular solutions of system (1.1). Theorem 1 is proved in Section 3. Finally, Theorem 2 is proved in Section 4.

\section{Particular solutions}

Proposition 1. The function (1.4) is a particular solution of system (1.3) if the homogeneous trigonometric polynomials $V_{k}(\varphi), k=1, \ldots, p$, satisfy the following differential system:

$$
\begin{aligned}
V_{k+1}^{\prime}+V_{k}^{\prime} Q_{s}+k(s-1) V_{k} P_{s} & =V_{k} V_{1}^{\prime}, \quad k=1, \ldots, p-1, \\
V_{p}^{\prime} Q_{s}+p(s-1) V_{p} P_{s} & =V_{p} V_{1}^{\prime} .
\end{aligned}
$$

where $^{\prime}=d / d \varphi$. 
Proof. If we force $V(R, \varphi)=0$ to be a particular solution of system (1.3), then it must satisfy

$$
\begin{aligned}
\dot{V}(R, \varphi) & =\lambda(R, \varphi) V(R, \varphi) \\
& =\lambda(R, \varphi)\left(1+V_{1}(\varphi) R+V_{2}(\varphi) R^{2}+\ldots+V_{p}(\varphi) R^{p}\right) .
\end{aligned}
$$

Differentiating $V(R, \varphi)$ with respect to $t$ we get

$$
\begin{aligned}
\dot{V}(R, \varphi)= & \frac{\partial V}{\partial R}\left((s-1) P_{s}(\varphi) R\right)+\frac{\partial V}{\partial \varphi}\left(1+Q_{s}(\varphi) R^{2}\right) \\
= & \left(V_{1}(\varphi)+2 V_{2}(\varphi) R+\ldots+p V_{p}(\varphi) R^{p-1}\right)\left((s-1) P_{s}(\varphi) R^{2}\right) \\
& +\left(V_{1}^{\prime}(\varphi) R+V_{2}^{\prime}(\varphi) R^{2}+\ldots+V_{p}^{\prime}(\varphi) R^{p}\right)\left(1+Q_{s}(\varphi) R\right) \\
= & V_{1}^{\prime}(\varphi) R+\sum_{k=1}^{p-1}\left(V_{k+1}^{\prime}(\varphi)\right. \\
& \left.+V_{k}^{\prime}(\varphi) Q_{s}(\varphi)+k(s-1) V_{k}(\varphi) P_{s}(\varphi)\right) R^{k+1} \\
& +\left(V_{p}^{\prime}(\varphi) Q_{s}(\varphi)+p(s-1) V_{p}(\varphi) P_{s}(\varphi)\right) R^{p+1}
\end{aligned}
$$

and if we equate the terms on the right-hand side of (2.2) and (2.3) it results first in $\lambda(R, \varphi)=V_{1}^{\prime}(\varphi) R$, and considering this relationship we obtain (2.1). We notice that $V(R, \varphi)$ satisfies $\dot{V}=\left(V_{1}^{\prime}(\varphi) R\right) V$.

Proposition 2. In order to find a particular solution of system (1.1) of the form (1.4) it is sufficient to find $p$ different solutions of the differential equation

$$
\left(x-Q_{s}(\varphi)\right) \frac{d x}{d \varphi}=(s-1) P_{s}(\varphi) x
$$

so that the functions $V_{k}(\varphi), k=1, \ldots, p$, obtained from relations (1.6) be homogeneous trigonometric polynomials of degree $k(s-1)$.

Proof. By using the functions $x_{i}$ introduced in (1.5) we define

$$
\begin{array}{r}
\text { (2.4) } \quad V_{0}^{i}=1, \quad V_{1}^{i}=\sum_{\substack{j=1 \\
j \neq i}}^{p} x_{j}, \quad V_{2}^{i}=\sum_{\substack{j, k=1 \\
j<k \\
j, k \neq i}}^{p} x_{j} x_{k}, \ldots \\
\ldots, \quad V_{p-1}^{i}=x_{1} x_{2} \ldots x_{i-1} x_{i+1} \ldots x_{p},
\end{array}
$$

for $i=1, \ldots, p$. From (1.5) and (2.4) we get

$$
V_{k}=\frac{1}{k} \sum_{i=1}^{p} V_{k-1}^{i} x_{i}, \quad V_{k}^{\prime}=\sum_{i=1}^{p} V_{k-1}^{i} x_{i}^{\prime}
$$


where $k=1, \ldots, p$. Then we can write system (1.5) as

$$
\begin{aligned}
& \sum_{i=1}^{p} V_{k}^{i} x_{i}^{\prime}+\left(\sum_{i=1}^{p} V_{k-1}^{i} x_{i}^{\prime}\right) Q_{s}+(s-1)\left(\sum_{i=1}^{p} V_{k-1}^{i} x_{i}\right) P_{s}=V_{k}\left(\sum_{i=1}^{p} x_{i}^{\prime}\right), \\
&\left(\sum_{i=1}^{p} V_{p-1}^{i} x_{i}^{\prime}\right) Q_{s}+(s-1)\left(\sum_{i=1}^{p} V_{p-1}^{i} x_{i}\right) P_{s}=V_{p}\left(\sum_{i=1}^{p} x_{i}^{\prime}\right),
\end{aligned}
$$

where $k=1, \ldots, p-1$. On the other hand, from the equalities

$$
\begin{gathered}
V_{k} \sum_{i=1}^{p} x_{i}^{\prime}-\sum_{i=1}^{p} V_{k}^{i} x_{i}^{\prime}=\sum_{i=1}^{p}\left(V_{k}-V_{k}^{i}\right) x_{i}^{\prime}=\sum_{i=1}^{p} V_{k-1}^{i} x_{i} x_{i}^{\prime}, \quad k=1, \ldots, p-1, \\
V_{p} \sum_{i=1}^{p} x_{i}^{\prime}=\sum_{i=1}^{p} V_{p} x_{i}^{\prime}=\sum_{i=1}^{p} V_{p-1}^{i} x_{i} x_{i}^{\prime},
\end{gathered}
$$

system (2.4) can be written as

$$
\begin{aligned}
& \sum_{i=1}^{p} V_{k-1}^{i}\left(x_{i}^{\prime} Q_{s}+(s-1) x_{i} P_{s}-x_{i} x_{i}^{\prime}\right)=0, \quad k=1, \ldots, p-1, \\
& \sum_{i=1}^{p} V_{p-1}^{i}\left(x_{i}^{\prime} Q_{s}+(s-1) x_{i} P_{s}-x_{i} x_{i}^{\prime}\right)=0 .
\end{aligned}
$$

If we set $X_{i}=x_{i}^{\prime} Q_{s}+(s-1) x_{i} P_{s}-x_{i} x_{i}^{\prime}, i=1, \ldots, p$, system (2.5) becomes

$$
\sum_{i=1}^{p} V_{k-1}^{i} X_{i}=0, \quad k=1, \ldots, p .
$$

This is a linear system of $p$ equations in the variables $X_{1}, \ldots, X_{p}$. The matrix $A$ of system (2.6) is given by

$$
A=\left(\begin{array}{cccc}
1 & 1 & \ldots & 1 \\
V_{1}^{1} & V_{1}^{2} & \ldots & V_{1}^{p} \\
\ldots & \ldots & \ldots & \ldots \\
V_{p-1}^{1} & V_{p-1}^{2} & \ldots & V_{p-1}^{p}
\end{array}\right),
$$

and a straightforward computation shows that

$$
\operatorname{det} A=\prod_{\substack{i, j=1 \\ i<j}}^{p}\left(x_{i}-x_{j}\right) .
$$

If we assume that the variables $x_{1}, x_{2}, \ldots, x_{p}$ are pairwise different, then $\operatorname{det} A \neq 0$. Therefore, the only possible solution of the linear system (2.6) is $X_{i}=0$ for $i=1, \ldots, p$, that is,

$$
x_{i}^{\prime} Q_{s}+(s-1) x_{i} P_{s}-x_{i} x_{i}^{\prime}=0, \quad i=1, \ldots, p .
$$

This completes the proof. 
We note that system (1.3) and the system of Proposition 2 are equivalent if we make the change of variable $R=-1 / x$.

3. Null divergence factors. If system (1.1) is written in polar coordinates (see $(1.2))$, then the function $(V(R, \varphi))^{\alpha}$ is a null divergence factor for system (1.1) if

$$
\frac{1}{r} \frac{\partial}{\partial r}\left(\frac{P_{s}(\varphi) r^{s+1}}{(V(R, \varphi))^{\alpha}}\right)+\frac{\partial}{\partial \varphi}\left(\frac{1+Q_{s}(\varphi) r^{s-1}}{(V(R, \varphi))^{\alpha}}\right)=0 .
$$

Now assume that the function $V(R, \varphi)$ is of the form given in (1.4) with $R=r^{s-1}$. Then if we develop the expression (3.1) with respect to the powers of $R$, we have

$$
\begin{aligned}
(s+1) P_{s}+Q_{s}^{\prime}-\alpha V_{1}^{\prime} & =0, \\
V_{k+1}^{\prime}+V_{k}^{\prime} Q_{s}+k(s-1) V_{k} P_{s} & =V_{k} V_{1}^{\prime}, \quad k=1, \ldots, p-1, \\
V_{p}^{\prime} Q_{s}+p(s-1) V_{p} P_{s} & =V_{p} V_{1}^{\prime} .
\end{aligned}
$$

System (3.2) coincides with system (1.5) except that the value of $V_{1}$ in system (3.2) is determined as a function of $P_{s}(\varphi), Q_{s}^{\prime}(\varphi)$ and $\alpha$.

Proof of Theorem 1. From (1.6), Proposition 2 and (2.5), system (3.2) takes the form

$$
x_{i}^{\prime}=\frac{(s-1) P_{s} x_{i}}{x_{i}-Q_{s}}, \quad i=1, \ldots, p,
$$

with the condition

$$
(s+1) P_{s}+Q_{s}^{\prime}-\alpha \sum_{i=1}^{p} x_{i}^{\prime}=0 .
$$

If we take $z=Q_{s}$ as independent variable instead of $\varphi$, then we have

$$
\frac{d x_{i}}{d \varphi}=\frac{d x_{i}}{d Q_{s}} \frac{d Q_{s}}{d \varphi}, \quad i=1, \ldots, p,
$$

and (3.4) goes over to

$$
(s+1) P_{s}+Q_{s}^{\prime}-\alpha Q_{s}^{\prime} \sum_{i=1}^{p} \frac{d x_{i}}{d z}=0,
$$

which gives

$$
P_{s}=\frac{1}{s+1}\left(\alpha \sum_{i=1}^{p} \frac{d x_{i}}{d z}-1\right) Q_{s}^{\prime} .
$$

By inserting the expression (3.5) in system (3.3), and considering the change 
of variable $z=Q_{s}$ we can write

$$
\frac{d x_{i}}{d z}=\frac{s-1}{s+1} \cdot \frac{\alpha \sum_{j=1}^{p} \frac{d x_{j}}{d z}-1}{x_{i}-z} x_{i}, \quad i=1, \ldots, p .
$$

Then, isolating $d x_{i} / d z, i=1, \ldots, p$, in the above system we get

$$
\frac{d x_{i}}{d z}=\frac{\prod_{\substack{j=1 \\ j \neq i}}^{p}\left(z-x_{j}\right)}{\frac{s+1}{s-1} \prod_{j=1}^{p}\left(z-x_{j}\right)+\alpha \sum_{j=1}^{p}\left(\prod_{\substack{k=1 \\ k \neq j}}^{p}\left(z-x_{k}\right)\right) x_{j}} x_{i}, \quad i=1, \ldots, p .
$$

If we divide the numerator and denominator of this fraction by the product $\prod_{j=1}^{p}\left(z-x_{j}\right)$, we obtain system (1.6).

Note that system (1.6) is symmetric with respect to the variables $x_{i}$, $i=1, \ldots, p$.

We want to find functions of the form

$$
U\left(x_{1}, \ldots, x_{p}, z\right) \equiv H\left(x_{1}, \ldots, x_{p}\right)+z G\left(x_{1}, \ldots, x_{p}\right)
$$

so that, for system (1.6), $d U / d z=0$.

Proposition 3. In order to find functions of the form (3.6) for system (1.6) it is sufficient to find solutions of the partial differential system

$$
\begin{aligned}
\frac{\partial H}{\partial x_{i}}+x_{i} \frac{\partial G}{\partial x_{i}}+\alpha G=0, \quad i & =1, \ldots, p, \\
\sum_{i=1}^{p} x_{i} \frac{\partial G}{\partial x_{i}}+\frac{s+1}{s-1} G & =0 .
\end{aligned}
$$

Proof. If we differentiate (3.6) with respect to $z$, we have

$$
\frac{d U}{d z}=\sum_{i=1}^{p} \frac{\partial H}{\partial x_{i}} \frac{d x_{i}}{d z}+z \sum_{i=1}^{p} \frac{\partial G}{d x_{i}} \frac{d x_{i}}{d z}+G=0 .
$$

By replacing the value of $d x_{i} / d z, i=1, \ldots, p$, given in (1.6) in the previous expression it becomes

$$
\sum_{i=1}^{p} \frac{\partial H}{\partial x_{i}} \frac{x_{i}}{z-x_{i}}+z \sum_{i=1}^{p} \frac{\partial G}{\partial x_{i}} \frac{x_{i}}{z-x_{i}}+\left(\frac{s+1}{s-1}+\alpha \sum_{i=1}^{p} \frac{x_{i}}{z-x_{i}}\right) G=0,
$$

or 


$$
\begin{gathered}
\sum_{i=1}^{p} \frac{\partial H}{\partial x_{i}} \frac{x_{i}}{z-x_{i}}+\sum_{i=1}^{p} \frac{\partial G}{\partial x_{i}}\left(x_{i}+\frac{x_{i}^{2}}{z-x_{i}}\right)+\left(\frac{s+1}{s-1}+\alpha \sum_{i=1}^{p} \frac{x_{i}}{z-x_{i}}\right) G \\
=\sum_{i=1}^{p} \frac{x_{i} \frac{\partial H}{\partial x_{i}}+x_{i}^{2} \frac{\partial G}{\partial x_{i}}+\alpha x_{i} G}{z-x_{i}}+\sum_{i=1}^{p} x_{i} \frac{\partial G}{\partial x_{i}}+\frac{s+1}{s-1} G=0 .
\end{gathered}
$$

In order that this last expression be null it is sufficient that conditions (3.7) hold. Notice that these conditions are not necessary in order that the previous expression be null.

4. Quadratic null divergence factors. We now consider the case $p=2$, that is to say,

$$
V(R, \varphi)=1+V_{1}(\varphi) R+V_{2}(\varphi) R^{2} .
$$

In this case, system (3.2) takes the form

$$
\begin{aligned}
(s+1) P_{s}+Q_{s}^{\prime}-\alpha V_{1}^{\prime} & =0, \\
V_{2}^{\prime}+V_{1}^{\prime} Q_{s}+(s-1) V_{1} P_{s} & =V_{1} V_{1}^{\prime}, \\
V_{2}^{\prime} Q_{s}+2(s-1) V_{2} P_{s} & =V_{2} V_{1}^{\prime},
\end{aligned}
$$

where $V_{1}=x_{1}+x_{2}, V_{2}=x_{1} x_{2}$, and system (1.7) goes over to

$$
\begin{aligned}
\frac{d x_{1}}{d z} & =\frac{\frac{x_{1}}{z-x_{1}}}{a+\alpha\left(\frac{x_{1}}{z-x_{1}}+\frac{x_{2}}{z-x_{2}}\right)}, \\
\frac{d x_{2}}{d z} & =\frac{\frac{x_{2}}{z-x_{2}}}{a+\alpha\left(\frac{x_{1}}{z-x_{1}}+\frac{x_{2}}{z-x_{2}}\right)},
\end{aligned}
$$

with $a=(s+1) /(s-1)$.

In this case, we want to obtain functions of the form

$$
U\left(x_{1}, x_{2}, z\right)=H\left(x_{1}, x_{2}\right)+z G\left(x_{1}, x_{2}\right)
$$

so that, for system (4.1), $d U / d z=0$.

By applying Proposition 3, the functions $H\left(x_{1}, x_{2}\right)$ and $G\left(x_{1}, x_{2}\right)$ have to satisfy the system

$$
\begin{array}{r}
\frac{\partial H}{\partial x_{1}}+x_{1} \frac{\partial G}{\partial x_{1}}+\alpha G=0, \\
\frac{\partial H}{\partial x_{2}}+x_{2} \frac{\partial G}{\partial x_{2}}+\alpha G=0, \\
x_{1} \frac{\partial G}{\partial x_{1}}+x_{2} \frac{\partial G}{\partial x_{2}}+a G=0 .
\end{array}
$$


If we make the change of variable $u=x_{2} / x_{1}$ and we take the functions $G$ and $H$ as follows:

$$
G\left(x_{1}, x_{2}\right)=x_{1}^{-a} g(u), \quad H\left(x_{1}, x_{2}\right)=x_{1}^{1-a} h(u),
$$

then the third equation of system (4.3) is satisfied identically, and the system takes the form

$$
\begin{gathered}
\alpha g+u \frac{d g}{d u}+\frac{d h}{d u}=0 \\
(1-a) h=[(a-\alpha)-\alpha u] g+u(1-u) \frac{d g}{d u} .
\end{gathered}
$$

If we differentiate the second equation of (4.4) with respect to $u$, we have

$$
(1-a) \frac{d h}{d u}=-\alpha g+[(a-\alpha+1)-(\alpha+2) u] \frac{d g}{d u}+u(1-u) \frac{d^{2} g}{d u^{2}} .
$$

By replacing the value of $d h / d u$ obtained from the first equation of system (4.4) in the previous expression, we find

$$
u(1-u) \frac{d^{2} g}{d u^{2}}+[(1+a-\alpha)-(1+\alpha+a) u] \frac{d g}{d u}-a \alpha g=0 .
$$

The relation (4.5) is a hypergeometric second order linear differential equation. We will study it for the particular case $a-\alpha=1 / 2$. This relation is satisfied by certain integrable systems (1.1) in the quadratic case $s=2$.

Since $a-\alpha=1 / 2$, the equation (4.5) can be written as

$$
u(1-u) \frac{d^{2} g}{d u^{2}}+\left(\frac{3}{2}-\left(2 a+\frac{1}{2}\right) u\right) \frac{d g}{d u}-a\left(a-\frac{1}{2}\right) g=0 .
$$

The general solution of this equation is given by

$$
g(u)=u^{-1 / 2}\left[C_{1}(1+\sqrt{u})^{2(1-a)}+C_{2}(1-\sqrt{u})^{2(1-a)}\right],
$$

where $C_{1}$ and $C_{2}$ are arbitrary constants. For this $g(u)$ we have

$$
h(u)=C_{1}(1+\sqrt{u})^{2(1-a)}-C_{2}(1-\sqrt{u})^{2(1-a)} .
$$

By going back through the change of variables it is easy to see that

$$
\begin{aligned}
& G\left(x_{1}, x_{2}\right)=\left(x_{1} x_{2}\right)^{-1 / 2}\left(C_{1}\left(\sqrt{x_{1}}+\sqrt{x_{2}}\right)^{2(1-a)}+C_{2}\left(\sqrt{x_{1}}-\sqrt{x_{2}}\right)^{2(1-a)}\right), \\
& H\left(x_{1}, x_{2}\right)=C_{1}\left(\sqrt{x_{1}}+\sqrt{x_{2}}\right)^{2(1-a)}-C_{2}\left(\sqrt{x_{1}}-\sqrt{x_{2}}\right)^{2(1-a)} .
\end{aligned}
$$

Therefore

$$
\begin{aligned}
& U_{1}\left(x_{1}, x_{2}, z\right)=\left(\sqrt{x_{1}}+\sqrt{x_{2}}\right)^{2(1-a)}\left(1+z / \sqrt{x_{1} x_{2}}\right), \\
& U_{2}\left(x_{1}, x_{2}, z\right)=\left(\sqrt{x_{1}}-\sqrt{x_{2}}\right)^{2(1-a)}\left(1-z / \sqrt{x_{1} x_{2}}\right)
\end{aligned}
$$


are two independent functions of the form (4.2) for system (4.1), which we can write in the form

$$
\begin{aligned}
& U_{1}\left(x_{1}, x_{2}, z\right)=\left(x_{1}+x_{2}+2 \sqrt{x_{1} x_{2}}\right)^{1-a}\left(1+z / \sqrt{x_{1} x_{2}}\right), \\
& U_{2}\left(x_{1}, x_{2}, z\right)=\left(x_{1}+x_{2}-2 \sqrt{x_{1} x_{2}}\right)^{1-a}\left(1-z / \sqrt{x_{1} x_{2}}\right) .
\end{aligned}
$$

As $V_{1}=x_{1}+x_{2}, V_{2}=x_{1} x_{2}, z=Q_{s}$ and $a=(s+1) /(s-1)$ we can write

$$
\begin{aligned}
& U_{1}\left(V_{1}, V_{2}, z\right)=\left(V_{1}+2 \sqrt{V_{2}}\right)^{-2 /(s-1)}\left(1+Q_{s} / \sqrt{V_{2}}\right), \\
& U_{2}\left(V_{1}, V_{2}, z\right)=\left(V_{1}-2 \sqrt{V_{2}}\right)^{-2 /(s-1)}\left(1-Q_{s} / \sqrt{V_{2}}\right),
\end{aligned}
$$

that is,

$$
\begin{gathered}
\left(V_{1}+2 \sqrt{V_{2}}\right)^{-2(s-1)}\left(1+Q_{s} / \sqrt{V_{2}}\right)=K_{1}, \\
\left(V_{1}-2 \sqrt{V_{2}}\right)^{-2 /(s-1)}\left(1-Q_{s} / \sqrt{V_{2}}\right)=K_{2},
\end{gathered}
$$

where $K_{1}$ and $K_{2}$ are arbitrary constants.

Proof of Theorem 2. We can write system (4.5) as

$$
\begin{aligned}
& V_{1}+2 \sqrt{V_{2}}=\bar{K}_{1}\left(1+Q_{s} / \sqrt{V_{2}}\right)^{(s-1) / 2}, \\
& V_{1}-2 \sqrt{V_{2}}=\bar{K}_{2}\left(1-Q_{s} / \sqrt{V_{2}}\right)^{(s-1) / 2} .
\end{aligned}
$$

By multiplying the two equations, we have

$$
V_{1}^{2}-4 V_{2}=\bar{K}_{1} \bar{K}_{2}\left(1-Q_{s}^{2} / V_{2}\right)^{(s-1) / 2} .
$$

As $V_{1}$ and $V_{2}$ are homogeneous trigonometric polynomials of degrees $s-1$ and $2(s-1)$ respectively, the left-hand side of $(4.7)$ is a trigonometric polynomial of degree $2(s-1)$. So the right-hand side of (4.7) must have the same degree. In particular, $V_{2}$ is a divisor of $Q_{s}^{2}$. On the other hand, if we square the first equation of (4.6), and we develop the right-hand side of that equation according to the Newton binomial, and group the terms with or without the factors $\sqrt{V_{2}}$, we see that $\sqrt{V_{2}}$ is a homogeneous trigonometric polynomial of degree $s-1$, and a divisor of $Q_{s}$. Hence $X_{2}=Q_{s} / \sqrt{V_{2}}$ is a homogeneous trigonometric polynomial of degree $(s+1)-(s-1)=2$, and we can write system (4.6) as

$$
\begin{aligned}
& V_{1}+2 \sqrt{V_{2}}=\bar{K}_{1}\left(1+X_{2}\right)^{(s-1) / 2}, \\
& V_{1}-2 \sqrt{V_{2}}=\bar{K}_{2}\left(1-X_{2}\right)^{(s-1) / 2} .
\end{aligned}
$$

By subtracting both equations of system (4.8), we have

$$
4 \sqrt{V_{2}}=\bar{K}_{1}\left(1+X_{2}\right)^{(s-1) / 2}-\bar{K}_{2}\left(1-X_{2}\right)^{(s-1) / 2},
$$

and then

$$
Q_{s}=X_{2} \sqrt{V_{2}}=\frac{1}{4} X_{2}\left(\bar{K}_{1}\left(1+X_{2}\right)^{(s-1) / 2}-\bar{K}_{2}\left(1-X_{2}\right)^{(s-1) / 2}\right) .
$$


If $s$ is even, the trigonometric polynomials $1+X_{2}$ and $1-X_{2}$ must be the squares of first degree homogeneous trigonometric polynomials in order to satisfy system (4.8). In the case where $s$ is odd, this condition is not necessary, but we can also impose it. We can easily prove that

$$
X_{2}(\varphi)=\cos 2\left(\varphi+\varphi_{0}\right)=\cos 2 \omega
$$

where $\varphi_{0}$ is arbitrary; it follows that

$$
1+X_{2}=2 \cos ^{2} \omega, \quad 1-X_{2}=2 \sin ^{2} \omega,
$$

and

$$
Q_{s}(\varphi)=\left(k_{1} \cos ^{s-1} \omega-k_{2} \sin ^{s-1} \omega\right) \cos 2 \omega,
$$

where $k_{1}=\frac{1}{4} \bar{K}_{1} 2^{(s-1) / 2}, k_{2}=\frac{1}{4} \bar{K}_{2} 2^{(s-1) / 2}$. By inserting the values obtained in (4.9) into system (4.8) we have

$$
V_{1}+2 \sqrt{V_{2}}=4 k_{1} \cos ^{s-1} \omega, \quad V_{1}-2 \sqrt{V_{2}}=4 k_{2} \sin ^{s-1} \omega .
$$

Therefore we obtain

$$
V_{1}=2\left(k_{1} \cos ^{s-1} \omega+k_{2} \sin ^{s-1} \omega\right), \quad V_{2}=\left(k_{1} \cos ^{s-1} \omega-k_{2} \sin ^{s-1} \omega\right)^{2} .
$$

Finally, $P_{s}$ is obtained from the first equation of (3.2):

$$
\begin{aligned}
P_{s} & =\frac{1}{s+1}\left(-Q_{s}^{\prime}+\alpha V_{1}^{\prime}\right)=\frac{1}{s+1}\left(-Q_{s}^{\prime}+\frac{s+3}{2(s-1)} V_{1}^{\prime}\right) \\
& =2\left(-k_{1} \cos ^{s-2} \omega \sin ^{3} \omega+k_{2} \sin ^{s-2} \omega \cos ^{3} \omega\right) .
\end{aligned}
$$

This completes the proof of the theorem.

\section{References}

[1] N. N. Bautin, On the number of limit cycles which appear with the variation of coefficients from an equilibrium position of focus or center of type $(R)$, Mat. Sb. 30 (72) (1952), 181-196 (in Russian); English transl.: Amer. Math. Soc. Transl. 100 (1954), 397-413.

[2] J. Chavarriga, Integrable systems in the plane with a center type linear part, Appl. Math. (Warsaw) 22 (1994), 285-309.

[3] C. Li, Two problems of planar quadratic systems, Sci. Sinica Ser. A 26 (1983), $471-481$.

[4] N. G. Lloyd, Small amplitude limit cycles of polynomial differential equations, in: Lecture Notes in Math. 1032, Springer, 1983, 346-356.

[5] V. A. Lunkevich and K. S. Sibirskiŭ, Integrals of a general quadratic differential system in cases of a center, Differential Equations 18 (1982), 563-568.

[6] D. Schlomiuk, Algebraic and geometric aspects of the theory of polynomial vector fields, in: Bifurcations and Periodic Orbits of Vector Fields, Kluwer Acad. Publ., 1993, 429-467.

[7] S. Shi, A method of constructing cycles without contact around a weak focus, J. Differential Equations 41 (1981), 301-312. 
[8] H. Żołądek, On a certain generalization of Bautin's Theorem, preprint, Institute of Mathematics, University of Warsaw, 1991.

JAVIER CHAVARRIGA

DEPARTAMENT DE MATEMÀTICA

ESCOLA TÉCNICA SUPERIOR D'ENGINYERIA AGRÀRIA

UNIVERSITAT DE LLEIDA

AVDA. ALCALDE ROVIRA ROURE, 177

25006 LLEIDA, SPAIN

Received on 20.12.1994 